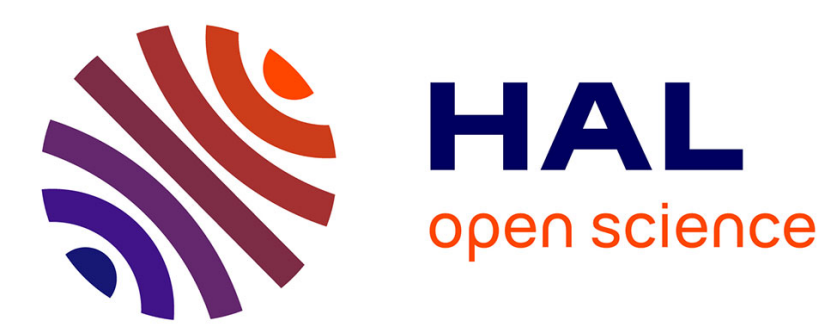

\title{
Probability Distributions of Local Modal-Density Fluctuations in an Electromagnetic Cavity
}

Andrea Cozza

\section{To cite this version:}

Andrea Cozza. Probability Distributions of Local Modal-Density Fluctuations in an Electromagnetic Cavity. IEEE Transactions on Electromagnetic Compatibility, 2012, 54 (5), pp.954-967. 10.1109/TEMC.2012.2190987 . hal-00673686

\section{HAL Id: hal-00673686 \\ https://hal-centralesupelec.archives-ouvertes.fr/hal-00673686}

Submitted on 24 Feb 2012

HAL is a multi-disciplinary open access archive for the deposit and dissemination of scientific research documents, whether they are published or not. The documents may come from teaching and research institutions in France or abroad, or from public or private research centers.
L'archive ouverte pluridisciplinaire HAL, est destinée au dépôt et à la diffusion de documents scientifiques de niveau recherche, publiés ou non, émanant des établissements d'enseignement et de recherche français ou étrangers, des laboratoires publics ou privés. 


\title{
Probability Distributions of Local Modal-Density Fluctuations in an Electromagnetic Cavity
}

\author{
Andrea Cozza, Member, IEEE
}

\begin{abstract}
Results from random-matrix theory are applied to the modeling of random fluctuations in the modal density observed in an electrically large cavity. By starting from results describing the probability distribution of the modal spacing between adjacent frequencies of resonance, or nearest-neighbor spacing, we introduce a simple procedure allowing to pass from the modal spacing to the local modal density as measured over a finite bandwidth. This local definition of the modal density is more consistent with the physics of reverberation chambers, since it has been recently shown that the deviation from asymptotic statistics of field samples is dependent on the number of modes overlapping within a modal bandwidth. It is shown that as opposed to current interpretation, the number of overlapping modes is a strongly fluctuating quantity, and that estimating it by taking the frequency derivative of Weyl's formula can lead to non-negligible errors and misunderstandings. Regarding these fluctuations as second-order effects is therefore not sound from a physical point of view, since the existence of modal depleted scenarios can easily explain the appearance of local anomalies in the field statistics, particularly, but not exclusively, in the lower frequency range of operation of reverberation chambers.
\end{abstract}

Index Terms-Cavities, mode-stirred reverberation chambers, stochastic fields, test facilities, field statistics, random-matrix theory, random fluctuations.

\begin{tabular}{|c|c|}
\hline & GLOSSARY \\
\hline$N(f)$ & $\begin{array}{l}\text { Cumulative number of modes up to the fre- } \\
\text { quency } f \text {. }\end{array}$ \\
\hline$N_{\mathrm{W}}(f)$ & Weyl's smooth approximation of $N(f)$. \\
\hline$N_{\mathrm{f}}(f)$ & $\begin{array}{l}\text { Residual fluctuations } N(f)-N_{\mathrm{W}}(f) \text { not ac- } \\
\text { counted for by Weyl's approximation. }\end{array}$ \\
\hline$m_{\mathrm{W}}(f)$ & $\begin{array}{l}\text { First derivative of } N_{\mathrm{W}}(f) \text {, used as an estimate } \\
\text { of the modal density. }\end{array}$ \\
\hline$M_{\mathrm{W}}(f)$ & $\begin{array}{l}\text { Average number of modes overlapping in a } \\
\text { bandwidth } B \text {, estimated as } B m_{\mathrm{W}}(f) \text {. }\end{array}$ \\
\hline$M_{\mathrm{loc}}(f)$ & $\begin{array}{l}\text { Actual number of modes overlapping in a } \\
\text { bandwidth } B \text {. }\end{array}$ \\
\hline$m_{\mathrm{loc}}(f)$ & $\begin{array}{l}\text { Homogenized local modal } \\
M_{\text {loc }}(f) / B \text {. }\end{array}$ \\
\hline$\varsigma_{W}^{2}(f)$ & $\begin{array}{l}\text { Normalized variance } \sigma^{2} / \mu^{2} \text { of the electric- } \\
\text { energy density } W \text {. }\end{array}$ \\
\hline
\end{tabular}

\section{INTRODUCTION}

$\mathbf{T}$ HE prediction of the performance of mode-stirred (or tuned) reverberation chambers (MSRCs) as generators of random electromagnetic test scenarios is a fundamental topic both from a theoretical and practical point of view in the operation of these facilities. The main issue here is the

A. Cozza is with the Département de Recherche en Électromagnétisme, Laboratoire des Signaux et Systèmes (L2S), UMR 8506 SUPELEC - Univ Paris-Sud - CNRS, 3 rue Joliot-Curie, 91192 Gif-sur-Yvette, France. Contact e-mail: andrea.cozza@supelec.fr. reproducibility of tests carried out in them and in particular the need to ensure that the fields generated by any MSRC belong to the same type of probability law. The current understanding of MSRCs is that at suitably high frequencies the electric and magnetic fields can be accurately described as complex-valued (circular) Gaussian random variables.

Such a probability law is typically assumed as a reasonable choice due to the (expected) availability of a large number of normal modes at high frequencies [1], [2], [3], as opposed to what are regarded as undermoded scenarios, where alternative reference probability laws have been proposed [4], [5], [6].

Still, as recalled in [7], the Gaussian hypothesis is only but an approximate model and it is incapable to explain the appearance of frequencies at which the field statistics proves to deviate substantially from those of a Gaussian random variable. Excluding the existence of unconventional setup configurations where the excitation source is strongly coupled to the equipment under test (EUT) [8], experimental observations of local anomalies in field statistics, appearing as glitches, have been reported in several papers [9], [10], [11]: these phenomena, though partially tolerated in the current operation of MSRC [12], have not yet received a satisfying physical explanation. Anomalies of this kind usually imply statistical dispersions higher than expected for a diffuse field (perfect reverberation), taking the form of local deviations rather than systematic ones over a bandwidth: these are usually referred to as outliers [2], i.e., as samples not belonging to the reference law and suspected to indicate a problem of some sort in the setup.

A rather different explanation can be proposed as soon as we remember that modal representations of the electromagnetic field generated within a MSRC are accurately reproduced by considering a finite number of modes, and in particular the average number of modes $M_{\text {loc }}$ overlapping within the $-3 \mathrm{~dB}$ bandwidth of a mode [13]. Theoretical and experimental results presented in [13] proved that $M_{\mathrm{loc}}$ can be quite low (a few units) even at frequencies where a MSRC is regarded as fully functional: as a result, the hypothesis of a Gaussiandistributed field is no longer justified, and its use should be limited to an educated guess for approximate predictive models.

Our previous work in [13] proved that the standardized (or normalized) variance, or variability for simplicity, of the electric-energy density, can be predicted on the basis of a few macroscopic parameters, such as the frequency, the geometrical dimensions of the cavity and an estimate of its average quality factor. It was intended as a first step in a better understanding of anomalous field statistics, suggested as being basically due to a poor local modal overlapping, a 
fact already recognized [12], [6], without having been more deeply explored.

The accuracy of the modal density estimated from Weyl's formula is often taken for granted. In fact, as shown in this paper, this is not a sound approach, as the modal density should rather be treated as a random quantity, subjected to non-negligible random fluctuations. The apparent lack of any available model capable of predicting the likeliness of observing a strong reduction (or increase) in the local modal density makes any prediction of the probability of observing these phenomena practically impossible.

It is the aim of this paper to introduce the probability laws of the modal density as observed locally, over a finite bandwidth, an approach that is better matched to the concept of modal overlapping. The average number of modes and ultimately the local modal density are considered as random quantities, according to the concepts of random-matrix theory (RMT) [14], [15]. Our results are completely general and independent from the details of implementation of the MSRC, as they are based on universality classes, as defined in the context of RMT. Interestingly, the probability law of the local modal density is entirely characterized by the average modal density predicted by Weyl's formula and the class of statistics of the MSRC. A priori knowledge of the average quality factor, the volume of the cavity and the frequency of operation are thus sufficient to derive a complete description of the statistics of the local modal density.

The interest of these models is not merely relegated to a better physical understanding of MSRCs, but also has a direct impact on their practical use. The results here proposed can be invoked when studying how likely it is that the field statistics in a reverberation chamber deviates from the ideal case usually taken as a reference, by means of the procedure introduced in [13]: clearly, the model here proposed being derived on physical grounds, the probability of appearance of anomalous field statistics can be predicted without recurring to phenomenological approaches, such as those based on the idea of fitting empirical field distributions to general theoretical laws [11].

The paper is organized as follows: Section II discusses various definitions of modal density and overlapping, while summarizing some major results derived in the context of random-matrix theory at the basis of the derivation presented in the rest of this paper. Sections III and IV introduce auxiliary results later used in Section $\mathrm{V}$ in the derivation of the probability distributions of the local modal density. An empty cuboid cavity is used as a test case in Section VI, supporting our predictions of a strongly fluctuating local modal density. Some considerations about the practical impact of these results are presented in Section VII, with an emphasis on the concept of outliers and local anomalous field statistics. The Appendix presents a detailed general calculation of the number of overlapping modes that should be expected in a cavity, supporting our claim that weak modal overlapping should not be expected only in the lower frequency range, but even in what is usually expected to be the overmoded region.

\section{PRELIMINARY Discussions}

Our analysis takes its start from results already available in the literature: on the one hand the link between the variability of the energy density and the average number of overlapped modes observed at the working frequency, and on the other the statistics of modal-related quantities derived in the context of RMT. The purpose of this Section is to briefly recall these tools while emphasizing some physical concepts and limitations that play a fundamental role in the subsequent derivations.

\section{A. Local modal density and overlapping}

When thinking about the modal density, one intuitively associates it to a certain number of modes resonating around the working frequency. The modal density can therefore be defined as the average number $M_{B}$ of modes found in a bandwidth $B$,

$$
m_{B}(f)=\frac{M_{B}(f)}{B},
$$

and is therefore dependent on $B$ itself. As long as $B$ is large enough to encompass several modes, then (1) is an average that can be expected to converge to a single value, for $B$ large enough, predicted by Weyl's approximation [16]

$$
m_{\mathrm{W}}(f)=\frac{8 \pi V}{c_{0}^{3}} f^{2}+o(f)=\frac{8 \pi V_{\lambda}}{f}+o(f),
$$

with $V$ the volume of the cavity, $c_{0}$ the speed of light in the filling medium and $V_{\lambda}$ the volume measured in cubic wavelengths.

The definition (1) provides a more general framework than (2), since the modal density is considered in a local setting: for this reason, it will be referred to as the local modal density, associated to a specific bandwidth.

It is often practical to associate a specific value to the modal density $m(f)$, e.g., by taking the limit for $B \rightarrow 0$ : the discrete nature of the set of frequencies $\left\{f_{i}\right\}$ at which a cavity resonates implies that in practice $m(f)$ can only take two values, i.e., zero if no mode resonates at the working frequency $f$ or infinity otherwise [14], i.e.,

$$
m(f)=\lim _{B \rightarrow 0} m_{B}(f)=\sum_{i=1}^{\infty} \delta\left(f-f_{i}\right) .
$$

This outcome is inevitable as the distribution of the normal modes cannot approach the completeness of real numbers, thus leaving inevitable "gaps" between them.

The estimate $m_{\mathrm{W}}(f)$ is in general different from $m_{B}(f)$ because it is not derived as in (3), but in a less direct manner, by first introducing the function $N(f)$ describing the overall number of modes of a cavity up to the frequency $f$

$$
N(f)=\#\left\{f_{i}: f_{i} \leqslant f\right\},
$$

with \# the cardinality of a set. This function can be represented as the sum of a smooth approximation $N_{\mathrm{W}}(f)$ and a fluctuating function $N_{\mathrm{f}}(f)$ with zero average value

$$
N(f)=N_{\mathrm{W}}(f)+N_{\mathrm{f}}(f) .
$$


This smooth approximation was first derived by Weyl and was intended to provide an approximate solution asymptotically exact at infinite frequency [16]. The fact that the intensity of the residual fluctuations grows less quickly than $N_{\mathrm{W}}(f)$ as $f \rightarrow \infty$, thus ensuring

$$
\lim _{f \rightarrow \infty}\left|\frac{N_{\mathrm{f}}(f)}{N_{\mathrm{W}}(f)}\right|=0,
$$

should not be mistaken for an indication that modal density can be defined as often done, by taking the derivative of $N_{\mathrm{W}}(f)$ at the working frequency $f$, leading to the approximation

$$
m(f)=\lim _{B \rightarrow 0} \frac{M_{B}}{B} \cong \frac{\mathrm{d} N_{\mathrm{W}}(f)}{\mathrm{d} f}=m_{\mathrm{W}}(f) .
$$

As a matter of fact, the residual $R_{m}(f)=\mid m_{\mathrm{W}}(f)-$ $M_{B} / B \mid$ does not converge to zero, since $N_{\mathrm{f}}(f)$ takes on the discrete nature of $N(f)$, thus preserving the results in (3). It could be expected that the accuracy of the approximation (7) improves as the frequency, and thus $N(f)$, increases, hence leading to modes getting close enough to provide a sort of approximate continuity. Unfortunately, this is not the case, as well witnessed by the number variance, a measure of the intensity of the fluctuations of the modal density $m(f)$ around a smooth approximate, e.g., $m_{\mathrm{W}}(f)$, as it will be recalled in Section II-B. Not only fluctuations do not vanish with the frequency, but they actually increase in absolute intensity, though their relative intensity decreases, as proven by studying the number variance, a measure of modal fluctuations discussed in Section II-B. A practical example is given in Section VII for a cuboid cavity.

As it will be shown in the rest of this paper, these fluctuations cannot be dismissed as minor approximation errors, particularly when the average number of overlapped modes is not high enough, as happens to be the case even at frequencies well above the lowest usable frequency (LUF) as usually defined by thumb rules proposed in practice within the framework of EMC tests [12] (see the Appendix for more details).

The differences between $m(f)$ and $m_{\mathrm{W}}(f)$ play a central role when studying the average local modal overlapping $M_{\text {loc }}(f)$. This quantity represents the average number of modes found within a bandwidth $B_{M}$ equal to the average $-3 \mathrm{~dB}$ width of a mode, i.e., $B_{M}=f / \bar{Q}$, hence

$$
M_{\mathrm{loc}}(f)=m_{B_{M}}(f) \frac{f}{\bar{Q}(f)},
$$

with $\bar{Q}(f)$ the ensemble-average composite quality factor of a MSRC; the use of ensemble ${ }^{\dagger}$ averages will be indicated by means of an overhead bar.

As proven in [13], a high modal density in itself is not a guarantee of a diffuse field, ensuring Gaussian-distributed scalar field components; the dominant parameter is rather $M_{\text {loc }}(f)$, which is required to be $M_{\text {loc }}(f) \gg 1$ in order to support a diffuse field. Therefore, it makes more sense to directly

${ }^{\dagger}$ By this term we consider the ensemble of all the random realizations of cavities generated by varying boundary conditions, due to any stirring procedure, but sharing the same macroscopic properties, i.e., average quality factor, volume, average energy density, average modal density, etc. [6]. count the number of modes overlapping over $B_{M}$, rather than passing through (8), since it requires an estimate of the local modal density $m_{B_{M}}(f)$, as defined in (1). This subtle distinction makes all the difference and should not be underestimated: it could seem more natural to assume $m_{B_{M}}(f) \simeq m_{W}(f)$ and derive $M_{\text {loc }}(f)$ from (8), but in this way we would implicitly accept the notion of a deterministic and smoothly increasing modal density, with no random fluctuations, with a $m_{W}(f)$ not depending on $B_{M}$. On the other hand, it is tempting to just consider the average modal density (and overlapping), since in practice the ensemble-average of $m_{B_{M}}(f)$ can be quite close to $m_{W}(f)$; as discussed in Section VII, such an approximation directly leads to a fundamental misunderstanding about the origin of statistical anomalies, or outliers, originated by strong random fluctuations in the modal density expected for single realizations of the cavity.

When directly considering the number of modes overlapping over $B_{M}$, the corresponding modal density should rather be defined as in (1), with an implicit local definition depending on $B_{M}$. In practice, (1) is an average modal density, but in this context the average is not over the realizations (ensemble average), but rather over the bandwidth $B_{M}$ for a single realization. In other words, it represents a sort of locally homogenized modal density, spread equally over the entire modal bandwidth $B_{M}$ rather than as a set of singularities as in (3). For this reason, we will refer to it as a local average, in contrast to the ensemble average. It will be shown in Section VII that this apparently redundant distinction makes a big difference.

\section{B. Random matrix theory and universality classes}

Following these discussions, what is needed is a probabilistic description of the local modal density $m_{B_{M}}(f)$, as defined in (1). A theory answering to this need is provided in the next three Sections. The starting point is the probability distribution of the spacing between the frequencies of resonance of two adjacent modes, often referred to as nearest-neighbor spacing, as derived by means of RMT [14].

This short summary is certainly not intended to serve as an introduction to RMT, and the interested Reader may refer to the first three chapters in Stöckmann's seminal book [14]. Nonetheless, we will give a brief overview of the reasons why we can apply in practice the results derived in the context of quantum chaos to our problem of field statistics in modestirred reverberation chambers.

RMT was developed to deal with structures where a direct solution of Schrödinger equation is regarded as complex or simply ill-defined, e.g., when the Hamiltonian operator is unknown. This is the case for complex quantum structures, such as large nuclear compounds or mesoscopic structures (e.g., quantum dots). A solution to this type of problems was found by approximating the unknown Hamiltonian operator by means of a matrix, eventually of asymptotic infinite dimensions, whose entries are assumed to follow specific probability distributions [17]. This idea is directly related to a previous and very successful approach, namely statistical mechanics, where in a similar manner the problem of studying 
the (thermo)dynamics of a large collection of interacting particles was solved by considering a random description of the state variables of the particles. The drive in these approaches is not having a fine-level information of the system at the scale of the individual elements it is composed of: the focus is rather on its macroscopic behavior, described by means of statistical quantities related to the statistical moments of physical quantities of interest and in general by means of probability distribution functions.

RMT has been widely successful in this respect, and at least in its basic idea surprisingly simple; the same cannot be said for the mathematical details. The structural similarity existing between Helmholtz and Schrödinger equations has motivated studies comparing the results predicted by RMT to those observed in microwave experiments [18]. It is important to notice that a major difference between these two equations is the absence of an Hamiltonian operator in Helmholtz equation: the structure is the same, but the lack of an Hamiltonian hinders the drawing of a direct parallel between the two equations. It is for this reason that the application of RMT to cavities where classical waves (of any nature) propagate had virtually to wait for a fundamental piece of work, namely the BohigasGiannoni-Schmidt conjecture [19], where it was postulated that the results of RMT should apply to any complex system. A number of experimental validations have confirmed this conjecture, which is today widely accepted as a physical fact. Of particular interest for the EMC community are the works dealing with microwave cavities, i.e., unstirred reverberation chambers, where the accuracy of the prediction of RMT was proven beyond any doubt (e.g., [18]).

The rationale behind recalling these points is that the nomenclature used in RMT is somewhat cryptic, with definitions that make sense in the context of quantum chaos without having any correspondence in classical wave theory. The apparent validity of the Bohigas conjecture allowed a direct transfer of the RMT ideas from the former to the latter, hence the potentially confusing terminology.

In this framework, we need to recall that RMT is based on universality classes allowing to define fundamental symmetry properties of the random matrix approximation of the Hamiltonian, according to fundamental physical properties of the system under consideration, e.g., energy conservation, reciprocity, etc., independently from the fine details of the system. In this respect, we will consider two configurations of practical interest, the case of integrable systems, also referred to (improperly) as the Poissonian ensemble [20], and that of the Gaussian Orthogonal Ensemble (GOE) [14], characterized by time-reversal invariance, i.e., energy conservation. A precise definition of the first class is apparently not yet available outside the context of quantum chaos, but the analogy with microwave structures is still maintained. The important point to consider is that under the category of integrable systems is considered any system that do not present any trace of the features of wave-chaotic systems, in particular level repulsion and of course exponential sensitivity to initial conditions. In practice, the fact that frequencies of resonance can cross each other's path when a dynamical perturbation (stirring) is operating, is a direct measure of absence of a fully

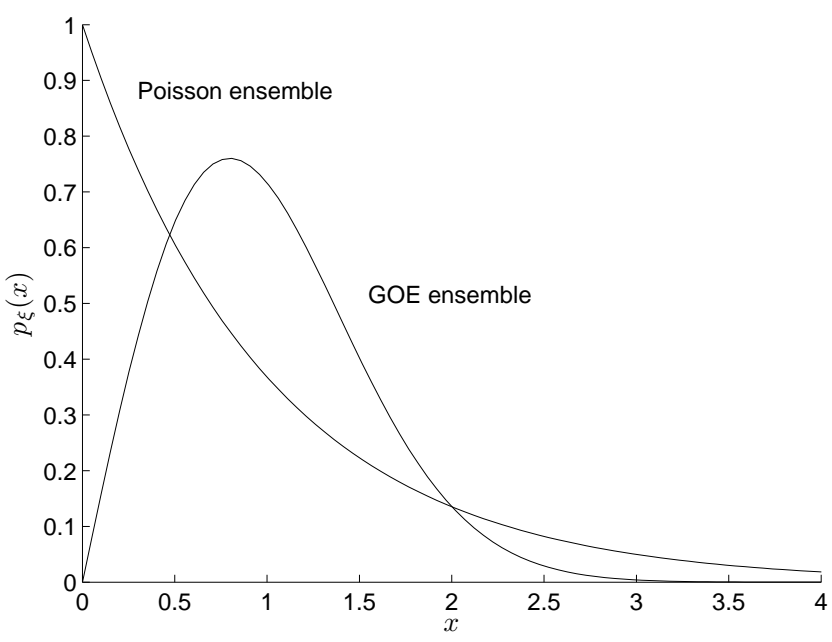

Fig. 1: Nearest-neighbor spacing probability density functions for an integrable and a GOE system, normalized to the ensemble-average spacing.

chaotic behavior. Integrable systems are actually regarded as an extreme case of non-chaotic systems, whereas in practice a certain amount of chaos is often observed [21]. In practice, completely empty rectangular cuboid cavities are a good example of integrable systems, while the inclusion of a scatterer spurs partially chaotic responses as soon as its dimensions are comparable to the wavelength. The GOE provides the other extreme representation for the ideal case of a fully chaotic system.

An example of direct interest for the EMC community was provided in [22], where it was shown through numerical simulations that a mechanical stirrer is not capable of providing a fully chaotic behavior, with traces of integrable features. It should be clear that the notion of integrable system is by no means related to the idea of degeneracy in the frequencies of resonance of a cavity, as in the case of an empty rectangular cavity with widths in rational proportion. Even in the case of irrational ratios, such a system will present the same behavior than any other integrable system.

The theory introduced in this paper is entirely based on the statistics of the nearest-neighbor spacing, defined as

$$
s_{i}=f_{i+1}-f_{i},
$$

where $s_{i}$ can be regarded, according to RMT, as the $i$-th realization of a random variable $s$, the probability density functions (pdfs) of the normalized nearest-neighbor spacing $\xi=s / \bar{s}$, with $\bar{s}=1 / m_{\mathrm{W}}$ the average nearest-neighbor spacing between adjacent modes, are [14]

$$
p_{\xi}(x)=\mathrm{e}^{-x},
$$

for a Poisson ensemble and

$$
p_{\xi}(x)=\frac{\pi}{2} x \mathrm{e}^{-\pi x^{2} / 4},
$$

for the GOE case. We are thus confronted to either an exponential distribution or a Rayleigh one with a parameter 


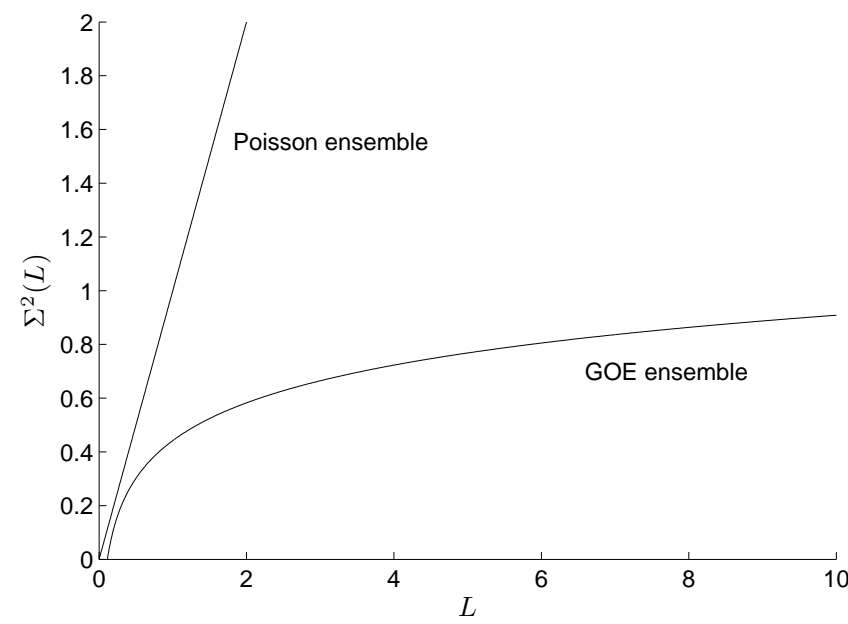

Fig. 2: Number variances $\Sigma^{2}(L)$ for an integrable and a GOE chaotic system.

$\sigma^{2}=2 / \pi$. These two functions are plotted in Fig. 1 where it is clear that the nil probability of superposed modes in chaotic systems is a direct consequence of level repulsion.

Two major differences can be noticed in these functions and will have a major impact on the statistics of the local modal density: 1) for chaotic systems, the modal spacing is decidedly less dispersed than for an integrable system, with a probability distribution presenting a mode (peak) close to the average spacing $\xi=1 ; 2$ ) for an integrable system, it is clear that modes can come in clusters due to a high probability of superposition [20], so that in order to maintain a fixed average spacing, the clusters must be relatively isolated one from the other, as justified by the longer exponential tail. We can refer to this phenomenon as modal depletion, i.e., the local lack of resonant modes, and it can be conjectured that the probability of incurring into what are often regarded as outliers [2] can be explained by this phenomenon. In other words, it is a natural and inevitable phenomenon in an integrable system, whereas it is to be expected less likely in chaotic systems.

According to the type of system we are dealing with, a higher probability of observing a wider nearest-neighbor spacing has a direct impact on the number of modes that can be observed in a fixed bandwidth, as will be derived in Section III.

As already recalled, practical systems are often in between these two extreme configurations, although a Poisson ensemble behavior should be expected in the lower frequency range when dealing with rectangular cavities: this result holds as long as eventual scatterers in the cavity are electrically small, after which the system moves gradually towards a chaotic one, as shown experimentally in [18]. Several methods have been devised to assess the degree of chaoticity of a cavity: in the context of this work, we will restrict our discussions and computations to the two extreme classes already introduced. The following results are directly applicable to the more general case of intermediary statistics for the modal nearestneighbor spacing.

A direct measure of the impact on the fluctuations of the modal density for the two universality classes can be obtained by studying their number variance $\Sigma^{2}(L)$, defined as the variance in the number of modes observed over a bandwidth containing on average $L$ modes, i.e., $L \bar{s}=L / m_{\mathrm{W}}$. The number variance is equal to

$$
\Sigma^{2}(L)=L,
$$

for an integrable system and

$$
\Sigma^{2}(L)=\frac{2}{\pi^{2}} \ln (2 \pi L)+0.0696+O\left(L^{-1}\right),
$$

for a GOE chaotic one, as proven in [15].

The number variance is a measure of the standard deviation of fluctuations in the modal density with respect to the average one defined by means of Weyl's formula. As made clear by Fig. 2, for an increasing $L$ the fluctuations can be quite severe for an integrable system, as opposed to a chaotic one. In particular, the fact that the variance in the number of modes increases with an increasing bandwidth is a direct proof of the non-convergent behavior of the approximate modal density (2). The increasing intensity of the fluctuations supports our claim that assuming the average modal density as an accurate and reliable measure of the availability of a large number of modes at high frequency is not correct. Modal depleted frequency bandwidths can pop out at any frequency leading to increased variability in the field statistics [13], even at frequencies above the usual LUF definitions.

Unfortunately, the number variance cannot be employed as a predictive tool in the study of the probability of observing anomalous field statistics, since it does not give any measure of the way fluctuations evolve for rare events, i.e., towards the tails of the pdf of the local modal density.

RMT is an asymptotical theory capable of accurately predicting the statistical properties of the spectrum of a system (here the frequencies of resonance of a cavity) as long as it admits a sufficiently large number of states. It should be clear that RMT cannot pretend to be exact when the electrical dimensions of a cavity become small, i.e., in its lower frequency range where it mainly behaves as a high quality factor resonator, allowing only a very limited number of resonances. Hence, RMT can be applied successfully even at frequencies below the LUF, since the modal density is typically high enough to justify a statistical description.

Other universality classes could be considered, such as the Gaussian unitary ensemble, or GUE, but it is of minor interest in practice, as it is useful only in the case of non-reciprocal systems. It could nevertheless find some applications in the case of the testing of devices with ferromagnetic properties or in general employing non-reciprocal materials. This case will not be addressed in this paper, but the procedures here developed are valid in any other type of nearest-neighbor spacing statistics and can be readily applied to any other universality class.

\section{BANDWIDTH COVERED BY $n$ MODES}

Access to the nearest-neighbor spacing probability distribution allows deriving that of the bandwidth covered by $n$ modes. Knowledge of the latter is instrumental in the computation of 

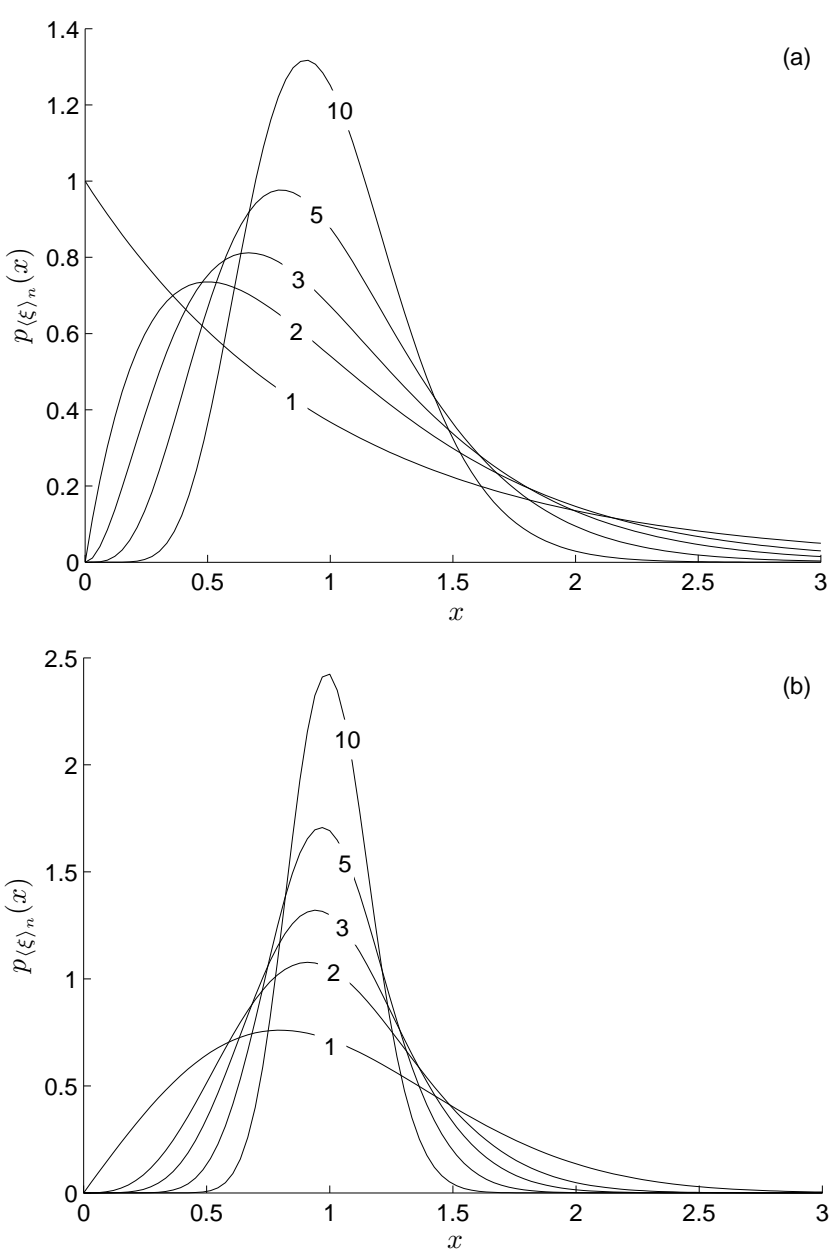

Fig. 3: Probability density functions of the normalized localaverage nearest-neighbor spacing $\langle\xi\rangle_{n}$, for (a) an integrable system and (b) a GOE chaotic one. Several values of $n$ are considered, showing the rate of convergence of the two universality classes towards the ensemble average value expected from Weyl's formula, corresponding to $x=1$.

the number of modes found within a fixed bandwidth and ultimately for the local modal density.

The total bandwidth $S_{n}$ covered by $n+1$ modes can be defined as

$$
S_{n}=\sum_{i=1}^{n} s_{i}=\frac{1}{m_{\mathrm{W}}} \sum_{i=1}^{n} \xi_{i},
$$

where the $n+1$ modes define $n$ random intervals or subbandwidths obeying to the parent laws introduced in the previous Section. We stick to the use of the normalized nearestneighbor spacing $\xi$, as this choice allows deriving completely general results. In this respect, it is better introducing the localaverage of the spacing of $n$ consecutive modes,

$$
\langle s\rangle_{n}=\frac{1}{n} \sum_{i=1}^{n} s_{i}=\frac{\langle\xi\rangle_{n}}{m_{\mathrm{W}}},
$$

where the normalized local average nearest-neighbor spacing $\langle\xi\rangle_{n}=\langle s\rangle_{n} / \bar{s}$ will have a central role in Section V.
The pdf $p_{\langle\xi\rangle_{n}}(x)$ implies carrying out $n$ convolutions of the original pdf of the random variable $\xi / n$,

$$
p_{\xi / n}(x)=n p_{\xi}(n x),
$$

since $\langle\xi\rangle_{n}$ involves the sum of $n$ such random variables that will be assumed to be iid. This procedure implies an approximation, as higher-order statistics, involving the mutual correlations between spacings at different distances is usually not identically equal to zero [14]. As the average modal density increases, with more packed resonances, the omission of their correlation can be expected to have an increasing importance.

In the case of an integrable system, where an exponential distribution is predicted for the nearest-neighbor spacing, the result of such operation is available in closed form and is the Gamma probability distribution, with $\langle\xi\rangle_{n} \in \Gamma(n, 1 / n)$. In the other cases, i.e., the GOE and any other intermediate nonfully chaotic system, no closed-form solution is available. A simple way of deriving the pdf of $\langle\xi\rangle_{n}$ is to pass through the characteristic function $\varphi_{\xi / n}(t)$ of $p_{\xi / n}(x)$ [23]

$$
\varphi_{\xi / n}(t)=\mathcal{F}\left\{p_{\xi / n}\right\}(t),
$$

by means of a Fourier transform. In the Fourier domain the $n$ convolutions correspond to

$$
\varphi_{\langle\xi\rangle_{n}}(t)=\left[\varphi_{\xi / n}(t)\right]^{n} .
$$

The pdf of the local-average normalized nearest-neighbor spacing can be retrieved by inverse-transforming its characteristic function

$$
p_{\langle\xi\rangle_{n}}(x)=\mathcal{F}^{-1}\left\{\varphi_{\langle\xi\rangle_{n}}\right\}(x),
$$

with the total random bandwidth covered by $n+1$ modes given by

$$
S_{n}=n \bar{s}\langle\xi\rangle_{n} .
$$

Some examples are given in Fig. 3, for the case of integrable and GOE systems. As expected for iid random variables, as $n$ increases, the central-limit theorem requires the pdf of their average to converge towards a bell-shaped function, asymptotically approaching a Gaussian function. What is important to notice is that the two groups of functions inherit the features of their respective parent law for the modal nearest-neighbor spacing. As a result, for the same ensemble-average modal nearest-neighbor spacing $\bar{s}=1 / m_{\mathrm{W}}$, the integrable case shows a sensibly larger statistical dispersion, with a much heavier tail for large nearest-neighbor spacings. Reciprocally, this implies that for a fixed bandwidth, the probability of finding a given number of modes should be expected to be smaller in the case of an integrable system than for a chaotic one. Again, this is related to the higher probability of close modes found in integrable systems, leading to clusters interleaved with modal depleted regions. A higher rate of modal-cluster formation is visible in Fig. 3(a), where the probability of finding $n$ modes packed into a bandwidth narrower than the average one is clearly higher than in the GOE case, especially for a small $n$. Although this could be 


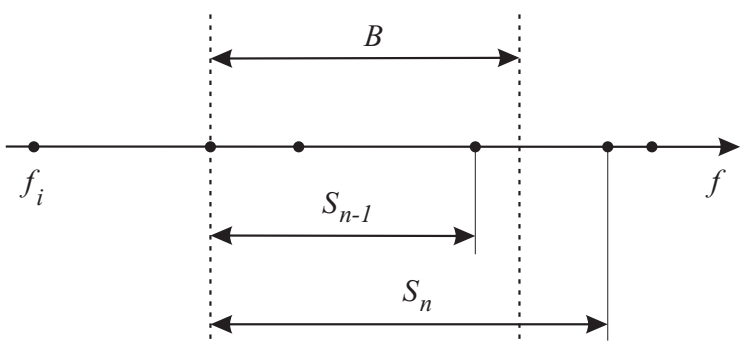

Fig. 4: Definitions of some modal-related quantities, showing the convention adopted in order to predict the number of modes appearing within $B$. Each dot represents a frequency of resonance of a cavity. The first resonance on the left of $B$ is taken as a reference in the count, and does not belong to $B$, being at its left.

interpreted as an advantage of integrable systems with respect to GOE ones, generating a higher modal density with nonnegligible probability, this comes with an also increased rate of depletion, as clear in the tail of the distributions.

\section{NUMber OF MOdES IN A FINITE BANDWIDTH}

The second element needed to derive the probability distribution of the local modal density is the probability law $p_{M}(n, B)$ of finding no more that $n$ modes within a fixed bandwidth $B$ (see Fig. 4). It can be derived straightforwardly by recalling that a bandwidth $B$ contains no more than $n$ modes if

$$
S_{n-1} \leqslant B<S_{n} .
$$

In order to provide an unambiguous procedure for counting these modes, the lower end of the bandwidth $B$ will be assumed to coincide with a resonance frequency, as shown in Fig. 4. Clearly, this definition provides a different count when other configurations are considered; in fact, this is not important, as the count is meant as an auxiliary parameter in the definition of the local modal density. The statistics of this latter is actually invariant with respect to translations along the frequency axis, since we are here talking about a fraction of the average spacing, so that our convention does not lead to any bias in the pdf of the local modal density. As a result of this choice, the first mode will not be counted as belonging to $B$, so that of the $n$ modes found in $B$, only $n-1$ will be counted. In other words, the first frequency of resonance is assumed to be on the left of $B$.

Hence, according to this convention, the probability law $p_{M}(n, B)$ is given by

$$
\begin{aligned}
p_{M}(n, B) & =P\left(\left\{S_{n+1}>B\right\} \cap\left\{S_{n} \leqslant B\right\}\right) \\
& =\int_{S_{n}=0}^{B} \int_{S_{n+1}=B}^{\infty} p\left(S_{n}, S_{n+1}\right) \mathrm{d} S_{n} \mathrm{~d} S_{n+1},
\end{aligned}
$$

where the joint pdf $p\left(S_{n}, S_{n+1}\right)$ is needed. It can be derived by expressing it as a function of conditional probabilities
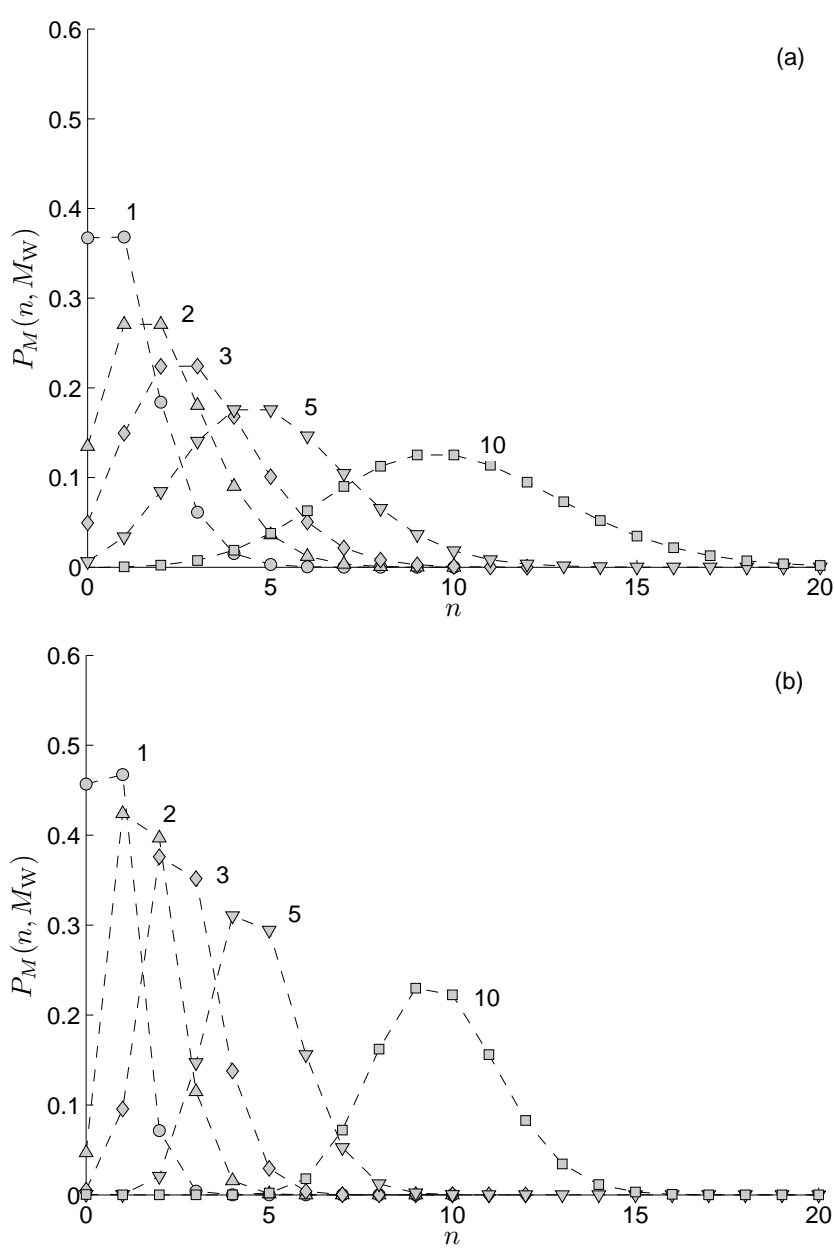

Fig. 5: Probability density functions $p_{M}\left(n, M_{\mathrm{W}}\right)$ for (a) an integrable system and (b) a GOE chaotic one for several values of the average number of modes $M_{\mathrm{W}}$. Notice the relatively high probability of finding no mode in the case of integrable systems with $M_{\mathrm{W}}<5$, with respect to the GOE case.

$$
\begin{aligned}
p\left(S_{n}, S_{n+1}\right) & =P\left(S_{n+1} \mid S_{n}\right) p_{S_{n}}\left(S_{n}\right) \\
& =p_{s}\left(S_{n+1}-S_{n}\right) p_{S_{n}}\left(S_{n}\right),
\end{aligned}
$$

where the conditional probability of observing a bandwidth $S_{n+1}$ covered by $n+2$ modes (counting the reference one) knowing that $n+1$ modes cover the bandwidth $S_{n}$ is actually equivalent to the probability of observing a further modal nearest-neighbor spacing $s=S_{n+1}-S_{n}$ between the last two modes. Hence

$$
\begin{aligned}
p_{M}(n, B) & =\int_{S_{n}=0}^{B} \int_{s=B-S_{n}}^{\infty} p_{s}(s) p_{S_{n}}\left(S_{n}\right) \mathrm{d} S_{n} \mathrm{~d} s \\
& =\int_{0}^{B} p_{S_{n}}(x)\left[1-F_{s}(B-x)\right] \mathrm{d} x,
\end{aligned}
$$

where $F_{s}(x)$ is the cumulative distribution function of the nearest-neighbor spacing $s$. Setting $y=x / \bar{s}$, this result can be recast as 


$$
p_{M}\left(n, M_{\mathrm{W}}\right)=\int_{0}^{M_{\mathrm{W}}} p_{\langle\xi\rangle_{n}}(y)\left[1-F_{\xi}\left(M_{\mathrm{W}}-y\right)\right] \mathrm{d} y,
$$

where

$$
M_{\mathrm{W}}=\frac{B}{\bar{s}}=m_{\mathrm{W}} B
$$

is the average number of modes expected over $B$ from an ensemble point of view. In the rest of this paper we will consider the case where $M_{\mathrm{W}}=m_{\mathrm{W}} B_{M}$, i.e., the average number of overlapped modes predicted by means of Weyl's formula (2).

Attention should be paid to the fact that the derivation of the probability function (25) is exact and applies for any number of modes, but for the case $n=0$. This case implies that the closest modes to the bandwidth are just outside it, i.e., the event (21) should now be substituted by the event $\{s>B\}$. Since (25) applies to any $n \in \mathbb{N} \backslash 0$, the normalization property of a pdf can be rather used to derive

$$
p_{M}\left(0, M_{\mathrm{W}}\right)=1-\sum_{i=1}^{\infty} p_{M}\left(i, M_{\mathrm{W}}\right) .
$$

Another property of (25) is that the average number of modes $\mathrm{E}[n]$ must coincide with that predicted by Weyl's formula, i.e., $M_{\mathrm{W}}$. This property has been numerically verified for the examples shown in Fig. 5.

The results obtained from (25) are shown in Fig. 5, where the increased statistical dispersion encountered for integrable systems is remarkably higher than for a chaotic one. Of particular interest is the non-negligible probability of observing no mode when $M_{\mathrm{W}}<5$ in an integrable system, i.e., of experiencing a modal depletion. This fact is important in practice, since an average overlapping $M_{\mathrm{W}}>5$ is not automatically achieved even at relatively high frequencies, as shown in the Appendix.

\section{LOCAL MODAL DENSITY DISTRIBUTIONS}

The local definition introduced in (1) can only account for an integer number of modes in $B_{M}$, whereas in practice we are rather interested in fractional values, too. With reference to Fig. 4, the local modal density can be defined as the ratio $n / S_{n}$, where $S_{n}$ is the bandwidth covered by $n+1$ modes (the first one being used as a reference), according to the definition (21). Hence the actual number of overlapping modes over a finite bandwidth, observed on a local scale, reads as

$$
M_{\mathrm{loc}}=\frac{n}{S_{n}} B_{M} .
$$

This definition is now capable of capturing all the intermediated cases where $B_{M}$ intercepts a fraction of the spacing separating two adjacent modes. For a given $M_{\text {loc }}$, an infinite number of modal scenarios can provide the same result. The distribution function $F_{M_{\mathrm{loc}}}(x)$ can now be computed by considering the entire set of events yielding the same equivalent modal overlapping over $B_{M}$, i.e.

$$
\begin{aligned}
\left\{M_{\mathrm{loc}}=x\right\} & =\bigcup_{n=1}^{\infty}\left\{\frac{n}{S_{n}} B_{M}=x\right\} \\
& =\bigcup_{n=1}^{\infty}\left\{\frac{n}{S_{n}} \bar{s}=\frac{x}{M_{W}}\right\} \\
& =\bigcup_{n=1}^{\infty}\left\{\langle\xi\rangle_{n}^{-1}=\frac{x}{M_{W}}\right\},
\end{aligned}
$$

recalling (15). Whence, partitioning the above event, we obtain

$$
\begin{aligned}
F_{M_{\mathrm{loc}}}(x) & =P\left(M_{\mathrm{loc}} \leqslant x\right)= \\
& =\sum_{n=1}^{\infty} P\left(\langle\xi\rangle_{n} \geqslant \frac{M_{W}}{x}\right) p_{M}\left(n, M_{\mathrm{W}}\right) \\
& =\sum_{n=1}^{\infty}\left[1-F_{\langle\xi\rangle_{n}}\left(\frac{M_{W}}{x}\right)\right] p_{M}\left(n, M_{\mathrm{W}}\right),
\end{aligned}
$$

with $M_{\mathrm{W}}$ defined by (26). Needless to say, since

$$
\frac{M_{\mathrm{loc}}}{M_{W}}=\frac{m_{\mathrm{loc}}}{m_{\mathrm{W}}},
$$

the same probability function holds also for the local modal density.

The pdf of $M_{\text {loc }}$ can then be straightforwardly retrieved by taking the derivative of (30) with respect to its argument. Expressing it in terms of the deviation from the overlapping $M_{\mathrm{W}}$ predicted by means of Weyl's formula yields

$$
p_{M_{\mathrm{loc}} / M_{\mathrm{W}}}(x)=x^{-2} \sum_{n=1}^{\infty} p_{\langle\xi\rangle_{n}}(1 / x) p_{M}\left(n, M_{\mathrm{W}}\right) .
$$

Some examples of this pdf are presented in Fig. 6: it is hence possible to assess the large domain of variability of the modal density, spanning more than one octave with a non negligible probability even at a relatively high modal overlapping of 10 modes, and up to two octaves for the integrable case.

The ensemble average $\bar{M}_{\text {loc }}$, mode and standard deviation of the local modal overlapping are shown in Fig. 7, as functions of $M_{\mathrm{W}}$. The mode and the ensemble average are on either side of the value predicted by Weyl's formula, indicating that although the modal density can be higher than expected for a weak modal overlapping, the most likely issue (mode) is lower.

The fact that the average local modal density is higher than predicted by Weyl's approximation (2) is a direct consequence of Jensen inequality [24], since the modal density is related to the nearest-neighbor spacing by means of a convex function.

In practice, a statistical mode systematically below the average implies that even with a GOE chaotic cavity the number of overlapping modes is likely lower than $M_{\mathrm{W}}$. The ensemble average for the case of an integrable system is much higher than expected, due to a strong skewness in the probability distributions in Fig. 6. Even more important is the fact that the standard deviation is still comparable with $M_{\mathrm{W}}$ even when $M_{\mathrm{W}} \gtrsim 10$, in both cases. This result implies that even a relatively strong modal overlapping is still affected 

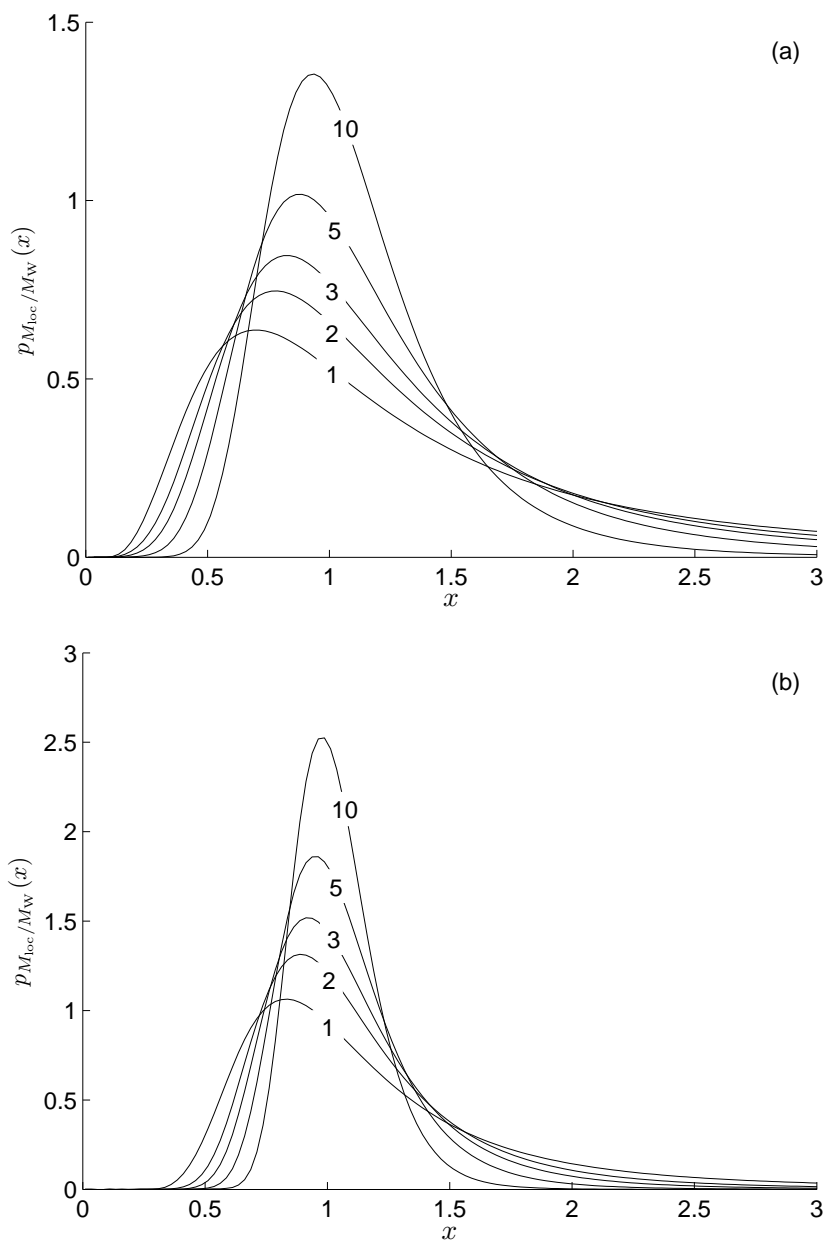

Fig. 6: Probability density functions of the local modal density, normalized to the ensemble-average modal density predicted by Weyl's formula, i.e., $M_{\mathrm{loc}} / M_{\mathrm{W}}$, for (a) an integrable system and (b) a GOE chaotic one.

by non-negligible random fluctuations, of the same order of magnitude as the average. Of course, their impact decreases with $M_{\mathrm{W}}$ : as discussed in [13], when $M_{\text {loc }} \gtrsim 3$, although the field will not be yet completely diffused, its deviation from the asymptotic statistics will become less sensitive to the actual number of overlapped modes. A much higher dependence from $M_{\text {loc }}$ is to be expected at a weak modal overlapping, where a large statistical dispersion can lead to a dramatic increase in the variability of the electric field.

These results, requiring no specific assumption on the fine details of the geometry of a cavity, give an insight into three important issues: 1) for a weak modal overlapping, the deviation of the field statistics predicted in [13] should be expected to present a strong statistical dispersion, and in particular a high probability of leading to even larger deviations than those predicted when using $M_{\mathrm{W}}$ as an estimate of the modal overlapping; 2) even at high frequencies where a large number of modes are expected to overlap, their actual number is still affected by a non-negligible statistical dispersion; 3) the differences between Poisson and GOE statistics vanish asymptotically for a large overlapping, e.g., mode and mean
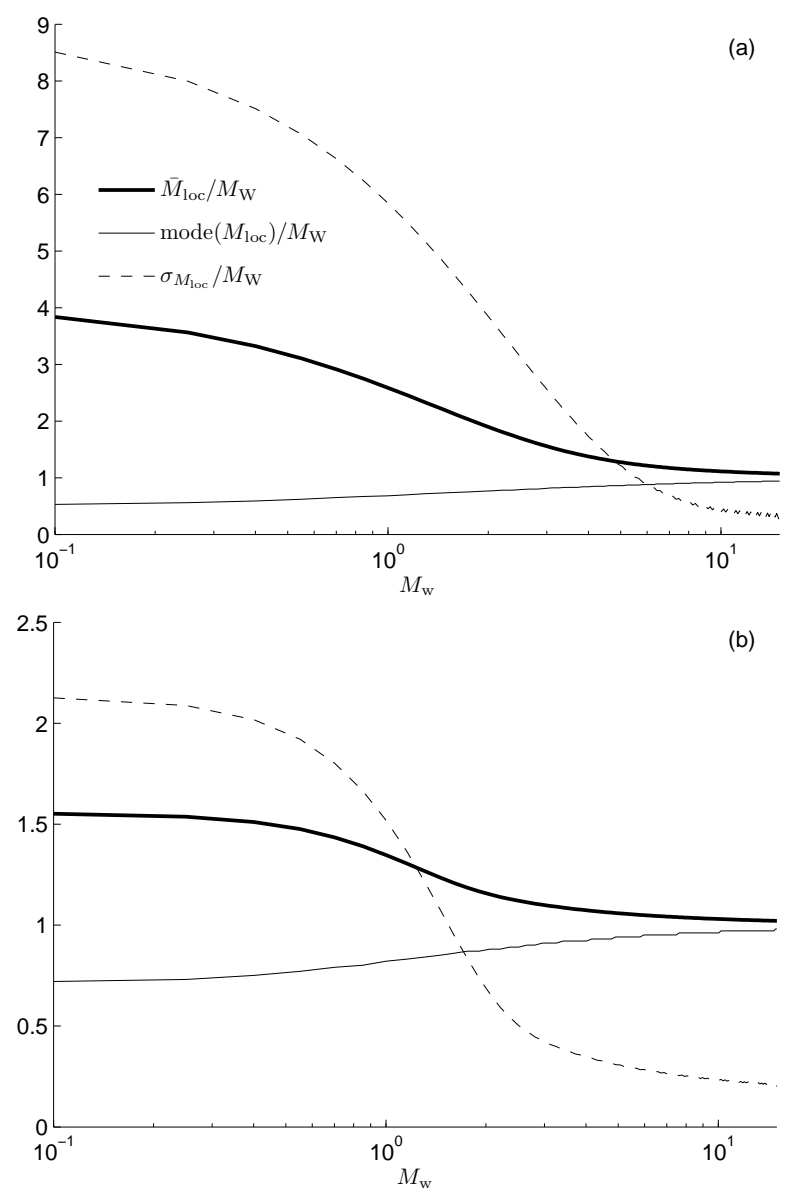

Fig. 7: Average value, mode and standard deviation of the local modal overlapping $M_{\text {loc }}$, normalized to the expected value $M_{\mathrm{W}}$ predicted by means of Weyl's formula, for (a) an integrable system and (b) a GOE chaotic one.

value converge to the same result, as expected by invoking the central-limit theorem. In particular for this last point, the standard deviation expected for the GOE case is about half of that in the integrable case.

We want to stress that these fluctuations in the field statistics must not be interpreted as non-compliancies or shortcomings of reverberation chambers, as they just belong to the normal range of physical responses expected for such systems. As made clear by Fig. 6, albeit the probability of experiencing a very low number of modes over $B_{M}$ decreases as $M_{W} \rightarrow \infty$, the probability is never equal to zero. In other words, it is unphysical to expect a reverberation chamber to present no anomalous statistics even at high frequency. We can conclude that the concept of outliers as suggested in [2] appears to originate from a biased interpretation of otherwise physically justified deviations in the field statistics generated by a reverberation chamber. While unlikely, these extreme scenarios due to mode-depleted frequencies are perfectly within the physiological response of a cavity.

\section{VALIDATION FOR A CUBOID CAVITY}

An experimental validation involving modal quantities is far from being a trivial task, since as soon as two consecutive 

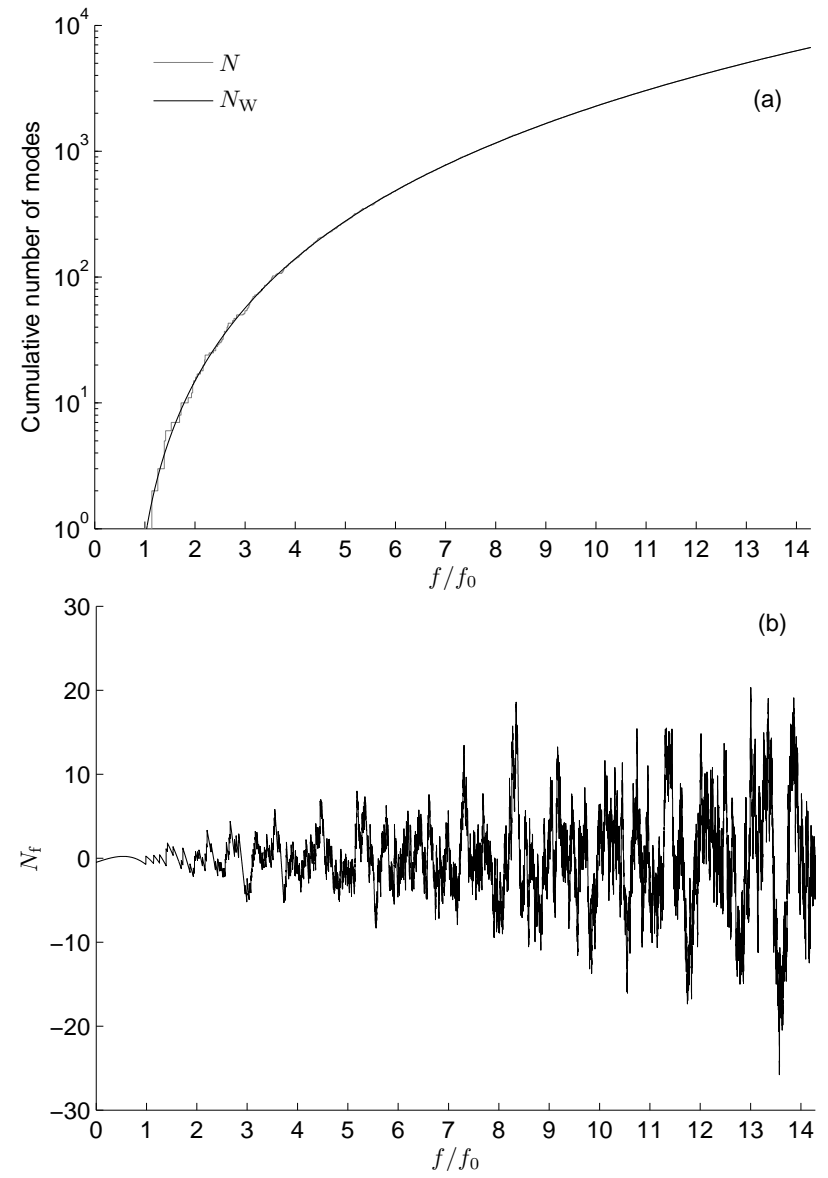

Fig. 8: Cumulative number of modes for the rectangular cuboid cavity taken as an example in Section VI: (a) comparison between the actual count $N(f)$ computed with (4) and (33) and Weyl's approximation (34); (b) the residual fluctuations $N_{\mathrm{f}}(f)$.

resonance frequencies are closer than the average modal bandwidth $B_{M}$, modal overlapping ensues making it hardly possible to distinguish and thus count the actual number of resonant frequencies.

A numerical validation is possible exclusively in the case of regular geometries, e.g., where Helmholtz equation can be solved by the method of separation of variables. A cavity in the shape of an empty rectangular cuboid is of practical interest within the framework of EMC test facilities, and will thus be taken as an example to illustrate the validity of our results.

A note of caution is nevertheless necessary, since the analysis of a regular geometry implies an integrable system, hence a Poisson class. As already pointed out, there is experimental evidence [18], [21], [22] that the behavior of real-life reverberation chambers is at least partially chaotic. Unfortunately, in this case no closed-form expression for the resonance frequencies is available.

For lateral dimensions $(a, b, c)$ of the cuboid cavity its frequencies of resonance can be computed by [25]

$$
f_{m n p}=\frac{c_{0}}{2} \sqrt{\left(\frac{m}{a}\right)^{2}+\left(\frac{n}{b}\right)^{2}+\left(\frac{p}{c}\right)^{2}},
$$

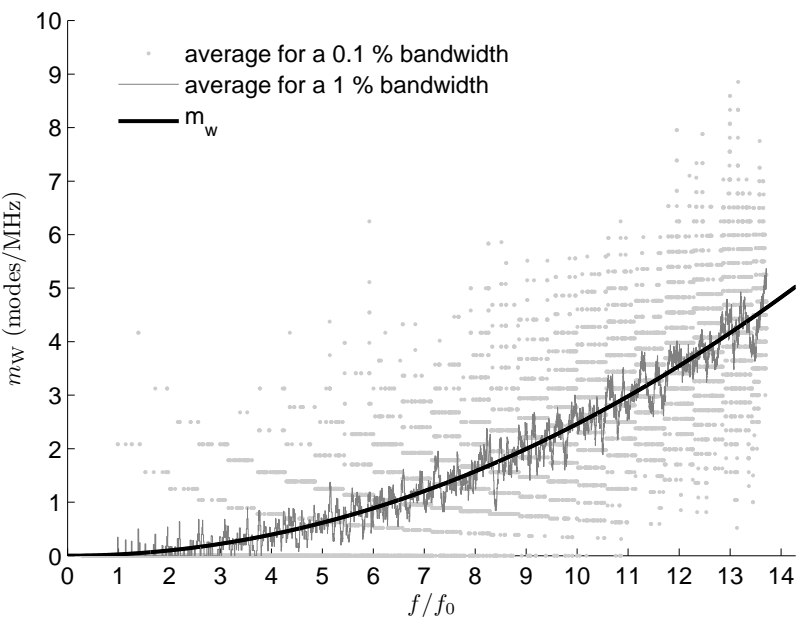

Fig. 9: Local modal density computed from $N(f)$, averaged over $0.1 \%$ and $1 \%$ relative bandwidths. The thick black curve is the result predicted by deriving Weyl's approximation (35). The relative bandwidth over which the average modal density should be computed is rather $1 / \bar{Q}$, which is usually much smaller than the $0.1 \%$ value here considered. Much stronger fluctuations should be expected in this case, making their graphical representation by far less clear.

It is therefore straightforward to compute the cumulative number of normal modes $N(f)$, by taking due care in counting in the degeneracies and allowed combinations of the triplet $(m, n, p)$ [25]. For the sake of providing a quantitative example, the choice $a=2.8 \mathrm{~m}, b=2.5 \mathrm{~m}, c=3.2 \mathrm{~m}$, corresponding to a volume $V=22.4 \mathrm{~m}^{3}$ and a fundamental resonance $f_{0}=71.2 \mathrm{MHz}$ will be considered throughout this Section. The resulting cumulative number of modes $N(f)$ is shown in Fig. 8(a).

A more accurate Weyl's approximation valid for the special case of an empty cuboid [25] will be used as a reference,

$$
N_{\mathrm{W}}(f)=\frac{8 \pi V_{\lambda}}{3}-\frac{(a+b+c)}{\lambda}+\frac{1}{2},
$$

predicting a modal density

$$
m_{\mathrm{W}}(f)=\frac{8 \pi V}{c_{0}^{3}} f^{2}-\frac{(a+b+c)}{c_{0}} .
$$

A comparison between the cumulative number of modes predicted by (34) and those obtained by directly counting them from (4) and (33) is shown in Fig. 8(a): the well-known ability of Weyl's approximation in accurately predicting the cumulative number of modes is retrieved. The residual error, i.e., the fluctuating part $N_{\mathrm{f}}(f)$ of $N(f)$ is shown in Fig. 8(b), where it is clear that $N_{\mathrm{f}}(f) \ll N(f)$, at least when $f / f_{0} \gtrsim 1$. Note how the intensity of the fluctuations increases with the frequency, as predicted by the number variance recalled in Section II-B.

Nevertheless, as already recalled in Section II, this should not be taken as a gauge of the accuracy of Weyl's approximation when dealing with modal densities. Fig. 9 shows a comparison between the results predicted by (35) and the 

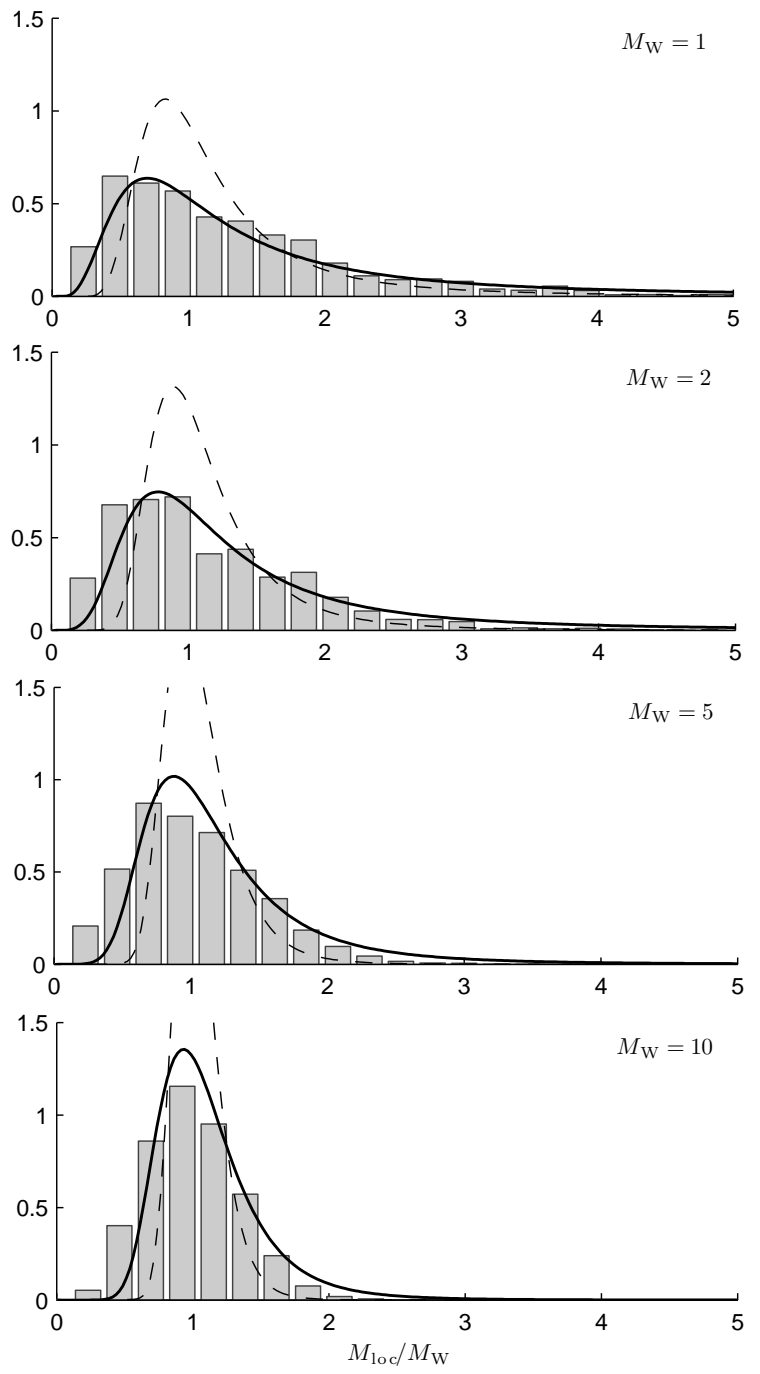

Fig. 10: Empirical probability distributions of the number of overlapped modes $M_{\text {loc }}$ observed for the rectangular cuboid cavity discussed in Section VI, obtained by observing 1000 bandwidths over the entire frequency range shown in Fig. 9. These results pertain to the local modal overlapping $M_{\text {loc }}$ counted over a frequency bandwidth where a reference overlapping $M_{\mathrm{W}}$ is predicted by means of Weyl's approximation (35). Four values of $M_{\mathrm{W}}$ are shown. The thick black curves represent the probability density functions predicted by our model and shown in Fig. 6(a) for an integrable cavity, while the dashed ones are for a GOE cavity, as given in Fig. 6(b).

actual local average modal density obtained over a relative bandwidth of $0.1 \%$ and $1 \%$ around a continuously varying frequency $f$. As discussed in Section II, the fluctuations are now far from negligible, with a high frequency of occurrence of regions of modal depletion, where even at relatively high frequency the modal density observed can be very close to zero.

The minimum frequency at which a cavity can be expected to be in an overmoded condition is often estimated at 5 to 10 times the fundamental resonance $f_{0}$. Fig. 9 proves that the actual average local modal density is still very strongly fluctuating around the value predicted by (35). It is noteworthy that the relative bandwidth over which the actual modal density should be averaged is equal to $1 / \bar{Q}$. Now, $\bar{Q}$ is never as low as just a few hundred units. Therefore, even the results computed over a $0.1 \%$ bandwidth are optimistic in their display of a strongly fluctuating local modal density, since the value of $1 / \bar{Q}$ should rather be expected into the $10^{-6}-10^{-4}$ range, with even wider fluctuations.

In order to validate our prediction about the pdf of the number of overlapping modes, we proceeded to a direct count based on the definition (28). The bandwidths over which this operation was carried out were computed by taking Weyl's approximation (35), imposing a given $M_{\mathrm{W}}$ and finding out the bandwidth $M_{\mathrm{W}} / m_{\mathrm{W}}(f)$ over which this number of modes should be expected to overlap at a given frequency. The four values $M_{\mathrm{W}}=\{1,2,5,10\}$ were considered, and the actual count $M_{\mathrm{loc}}(f)$ was computed over 1000 bandwidths distributed over the entire frequency range, starting at $f=$ $2 f_{0}$. The empirical probability distributions thus obtained are shown in Fig. 10(a)-(d), where they are compared to the pdfs shown in Fig. 6(a). The good agreement between these results prove that in practical configurations the actual number of overlapping modes can definitely be smaller than expected when using Weyl's approximation, even when a relatively high average modal overlapping is expected.

The question of what average modal overlapping should be expected in practice is treated in detail in the Appendix, where it is shown that a weak modal overlapping, i.e., $M_{\mathrm{W}}<3$, is far from unlikely. Experimental results pertaining to this issue have also been shown in [13].

\section{Practical Considerations}

As already recalled in Section I, these discussions about random fluctuations in the modal overlapping have a direct practical impact, because of the direct link existing between the average local modal density over $B_{M}$, and thus the modal overlapping, and the variability $\varsigma_{W}^{2}$ of the energy density $W=$ $\epsilon_{0}\|\boldsymbol{E}\|^{2}$, as measured at any position inside at least a subvolume of a MSRC, a region usually referred to as working volume, with

$$
\varsigma_{W}^{2}=\left(\frac{\sigma_{W}}{\bar{W}}\right)^{2}
$$

and $\sigma_{W}$ the standard deviation of $W$.

As demonstrated in the Appendix and already shown in [13], the number of overlapped modes actually intervening can be quite low even at frequencies above the LUF estimated by means of the usual thumb rules. Under such conditions, the variability of $W$ is bound to be higher than expected, as demonstrated in [13]

$$
\varsigma_{W}^{2}=\frac{1}{3}+\frac{2}{\pi M_{\mathrm{loc}}} .
$$

The ensemble-average modal density was considered in [13], with $m_{B_{M}}(f)$ in (8) approximated by its ensemble average, $\bar{m}_{B_{M}}(f) \simeq m_{\mathrm{W}}(f)$, thus neglecting the random fluctuations that inevitably affect it, as proven in the previous Sections. Having only access to the estimate of the modal density provided by Weyl's formula, only the average deviation 

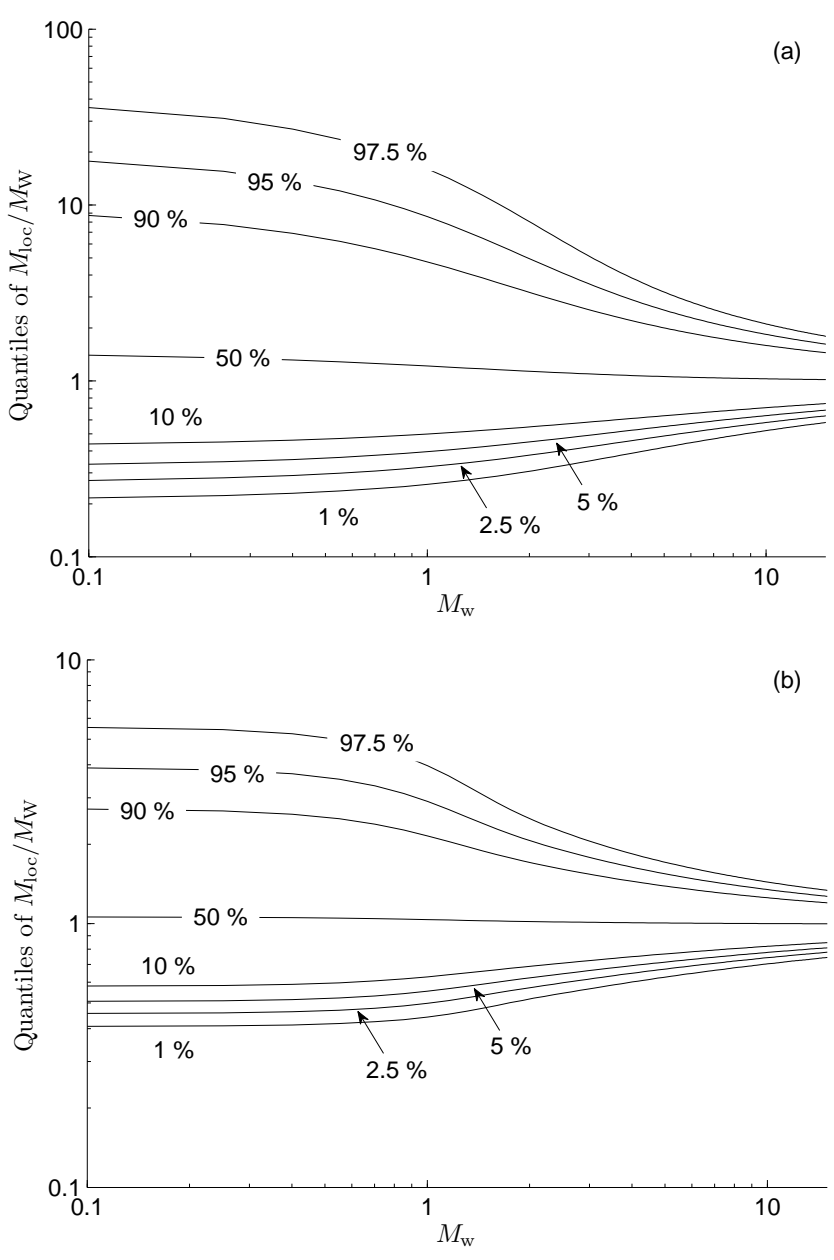

Fig. 11: Quantiles of the deviation of the local modal overlapping with respect to the estimate obtained from Weyl's formula, for (a) an integrable system and (b) a GOE chaotic one.

can be predicted, or an upper bound, as done in [13]. The non-negligible probability of observing a modal overlapping even weaker than expected has thus a direct and measurable impact on the statistics of the field generated by a reverberation chamber. As long as the actual number of overlapping modes $M_{\text {loc }} \gg 1$, this error can be entirely negligible, since (37) converges to the value $1 / 3$ expected for a diffuse field; but in the case of weak modal overlapping, as already discussed and proved in [13], very strong statistical deviations can ensue, particularly when $M_{\text {loc }} \lesssim 3$.

The following example should make this point clearer. In a cavity with an average modal overlapping $\bar{M}_{\text {loc }}=1$, (37) predicts an increase in the variability of $W$ equal to 0.63 , corresponding to a $191 \%$ relative deviation in the variability $\varsigma_{W}^{2}$. Of all the random realizations generated by a stirring technique, sharing the same average modal density, those presenting $M_{\mathrm{loc}}=1 / 2$ will be affected by an electric energy density with a statistical variability amplified by a factor 2 , i.e., about $380 \%$, which can easily explain anomalous field statistics on a local scale. As clear from Fig. 6, such an event is not unlikely. If $\bar{M}_{\text {loc }}=3$, than the relative deviation in the variability would rather pass from $63 \%$ to $126 \%$, if a realization featured half the average density. Therefore, depending on the average modal overlap, fluctuations can have a very different impact, with fields behaving with an increased statistical dispersion than expected from ideal reverberation models [2]. From this example it is clear that the strongest effect will be felt when $\bar{M}_{\text {loc }} \lesssim 3$.

A useful summary of the probability of occurrence of random fluctuations is given in Fig. 11, where the quantiles of the random variable $M_{\mathrm{loc}} / M_{\mathrm{W}}$ are computed for a varying $M_{\mathrm{W}}$. The median (50\% quantile) is very well approximated by the estimate $M_{\mathrm{W}}$ provided by Weyl's formula. Hence, there is an equal probability of observing either a higher or lower modal overlapping. In the context of deviations from the asymptotic statistics for field samples, the most important quantiles are those related to the probability of observing a lower modal overlapping. In this respect, when expecting $M_{\mathrm{W}}=1$, there is a $10 \%$ probability of observing an actual modal overlapping below $49 \%$ and $63 \%$ of $M_{\mathrm{W}}$ for an integrable and GOE cavity, respectively. Such strong reduction is proven by our derivation to be a normal phenomenon in a large cavity, and not related to any non-ideality in its use. A $50 \%$ reduction in the modal overlapping leads to a twofold increase in the additional term of the variability of the electric energy density, as demonstrated in [13] and recalled in (37). Worse, but perfectly normal, scenarios can appear : with a probability of $1 \%$ the modal overlapping can be found below $25 \%$ and $44 \%$ of $M_{\mathrm{W}}$. In other words, rare phenomena of very strong modal depletion can explain the existence of anomalous field statistics in a MSRC that is otherwise standard compliant.

These results could be expected to improve when a higher modal overlapping of $M_{\mathrm{W}}=3$ is considered. This value is often taken as a reference for the appearance of a diffuse-field condition in room acoustics [26]. Even in this case, $M_{\text {loc }}$ can be lower than $58 \%$ and $72 \%$, and with a probability of $1 \%$, below $34 \%$ and $56 \%$. Hence, even at relatively high modal overlappings, the probability of observing normal strong deviations in the field statistics should not be underestimated.

A probability of $1 \%$ is compatible with the rate of appearance of local non-compliancies as tolerated in current practice [12], and could thus provide a physical explanation to the observation of outliers [2]. It could also serve as an explanation for the existence of local non-compliancies even at higher frequencies, where the concept of overmoded cavity is usually taken for granted.

Of notable importance is the observation of a much higher statistical dispersiveness for an integrable case. In practice, this scenario is to be expected only when the scatterers within a reverberation chamber are no longer electrically large, i.e., towards their lower frequency range of operation, close to the LUF. It is thus pertinent to wonder if the inclusion of large passive scatterers within a chamber could improve at least the field statistics, by making the cavity chaotic rather than integrable. A similar idea was already vented in previous papers, but it was rather based on the hope of increasing the modal density [27], [28]. Our suggestion is of a different order: to reduce the statistical dispersion in modal overlapping 
by making a cavity chaotic, in order to avoid even stronger local deviations in the field statistics, due to modal depletions justified by stronger random fluctuations for the integrable case.

\section{CONCLUSIONS}

In this paper we have applied universal results from RMT in order to derive the probability distributions of modal-related quantities of interest to the physics of MSRC. These laws are entirely general and just require a handful of macroscopic parameters to be used in practice: the volume of the cavity $V$, its average composite quality factor $\bar{Q}$, etc. The two universality classes representing an integrable and a GOE chaotic system serve as extremes in the actual behavior of real-life MSRCs.

The rationale for our analysis is the link proven in [13] between the average number of overlapped modes over the average bandwidth of a mode, directly depending on the local modal density and the deviation in the parent law of field samples, as assessed by their increased variability.

It was shown that the estimate of local modal density, and thus modal overlapping, yielded by Weyl's formula has a far from negligible probability of overestimating the availability of resonant modes, particularly when modes are already scantly overlapped, i.e., for $M_{\mathrm{W}} \lesssim 3$. Interestingly, the statistical dispersion of the modal density appears to be non-negligible even at frequencies where a relatively large number of modes is already overlapping, on average.

The inevitable consequence is the appearance of large deviations from the asymptotic Gaussian behavior expected for the field generated in an overmoded reverberation chamber. These results are expected to be the basis for a better understanding of anomalous field statistics; moreover, the fact of being firmly based on physical grounds makes them appealing in the study of the links existing between the physics of large cavities and the statistical properties of real-life reverberation chambers.

\section{ACKNOWLEDGEMENTS}

The Author is grateful to the anonymous Reviewers for their constructive remarks and suggestions.

\section{APPENDIX}

\section{AVERAGE MODAL OVERLAPPING $M_{\mathrm{W}}$ FROM WEYL'S APPROXIMATION}

The average number $M_{\mathrm{W}}$ of overlapping modes in a cavity can be estimated by means of Weyl's approximation. Since we are mainly interested in knowing the order of magnitude of $M_{\mathrm{W}}$, we will consider the basic approximation (2), yielding

$$
M_{\mathrm{W}}(f)=m_{\mathrm{W}}(f) \frac{f}{\bar{Q}}=\frac{8 \pi V_{\lambda}}{\bar{Q}} .
$$

The average composite quality factor $\bar{Q}$ can be expressed as the harmonic sum of the three main loss/leakage mechanisms [29]

$$
\frac{1}{\bar{Q}}=\sum_{i=1}^{3} \frac{1}{\bar{Q}_{i}}
$$

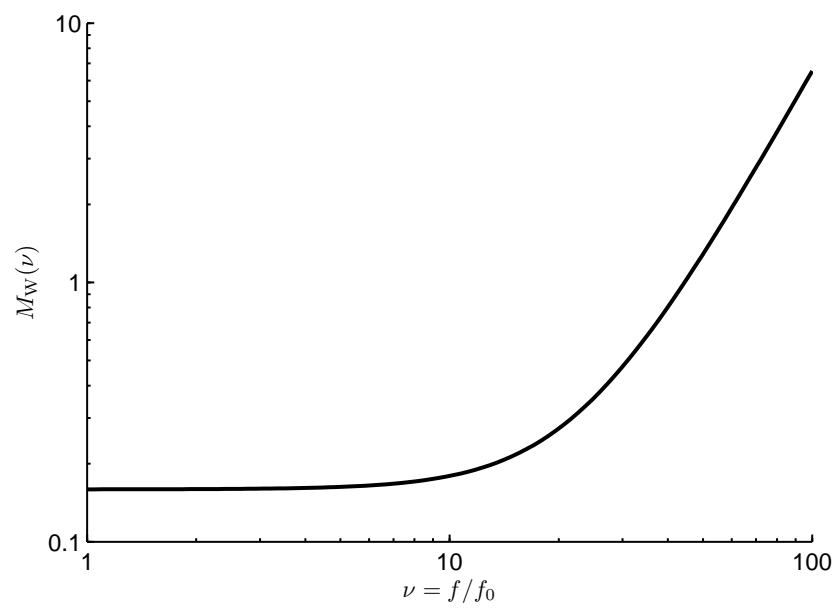

Fig. 12: Average modal overlapping predicted by (47) for dissipation in antenna loads and non-perfectly conductive metallic boundaries, neglecting the inclusion of additional lossy material into a cavity. These results refer to the case of a fundamental resonance at $20 \mathrm{MHz}$.

with

$$
\begin{aligned}
\bar{Q}_{1} & =16 \pi^{2} V_{\lambda} \\
\bar{Q}_{2} & =\frac{3 V}{2 \delta \mu_{w} S} \\
\bar{Q}_{3} & =\frac{2 \pi V}{\lambda \bar{\sigma}_{\mathrm{eq}}}
\end{aligned}
$$

where $V$ is the volume of the cavity and $S$ the surface of its metallic boundary.

$\bar{Q}_{1}$ models the dissipation in the antenna load (single antenna, here), for the special case of a perfectly matched antenna; $\bar{Q}_{2}$ represents Joule dissipation over imperfectly conductive walls, with $\mu_{w}$ the relative magnetic permeability of the metal covering the cavity surface and $\delta$ its effective skin-depth; $\bar{Q}_{3}$ accounts for power loss due to leakage through the cavity surface and dissipation in lossy materials within the cavity (e.g., absorbers) through an average absorption cross section, since they essentially behave in the same manner. We obtain from (38) - (40)

$$
M_{\mathrm{W}}(f)=\frac{1}{2 \pi}+4 \frac{\bar{\sigma}_{\mathrm{eq}}}{\lambda^{2}}+\frac{16 \pi}{3} \mu_{w} \frac{S \delta}{\lambda^{3}} .
$$

In the lower frequency range, the dominant term in (41) is $\bar{Q}_{1}$, i.e., dissipation in antenna loads. In this case

$$
\lim _{f \rightarrow 0} M_{\mathrm{W}}(f)=\frac{1}{2 \pi} \simeq 0.16,
$$

a result well below the average overlapping of 3 modes that is often regarded as ensuring a diffuse-field regime in a reverberating cavity [26].

In order to derive a simple closed-form expression, we will consider a cubic cavity, with side $a$ and non-magnetic metal surfaces, i.e., $\mu_{w}=1$. In this case the fundamental resonance frequency is

$$
f_{0}=\frac{c_{0}}{\sqrt{2} a}
$$


Expressing the frequency in terms of $f_{0}$, as $f=\nu f_{0}$, the wavelength becomes $\lambda=\lambda_{0} / \nu$, where $\lambda_{0}=\sqrt{2} a$. Hence (41) can be recast into

$$
M_{\mathrm{W}}(\nu)=\frac{1}{2 \pi}+4 \frac{\bar{\sigma}_{\mathrm{eq}}}{\lambda_{0}^{2}} \nu^{2}+\frac{16 \pi}{3} \frac{S \delta}{\lambda_{0}^{3}} \nu^{3} .
$$

The last term includes the skin depth, which is frequency dependent. A simplification is possible by writing

$$
\frac{S \delta}{\lambda^{3}}=\frac{\nu^{2.5}}{\sqrt{\lambda_{0}}} \frac{3}{\sqrt{\pi \mu_{0} \sigma_{w} c_{0}}},
$$

where $\sigma_{w}$ is the conductivity of the metallic surfaces. For a conductivity $\sigma_{w}=3.5 \cdot 10^{7} \mathrm{~S} / \mathrm{m}$ (aluminium), (44) becomes

$$
M_{\mathrm{W}}(\nu)=\frac{1}{2 \pi}+4 \frac{\bar{\sigma}_{\mathrm{eq}}}{\lambda_{0}^{2}} \nu^{2}+2.47 \cdot 10^{-4} \frac{\nu^{2.5}}{\sqrt{\lambda_{0}}} .
$$

We are now in condition to assess the average number of overlapping modes predicted by Weyl's approximation. For a cavity with $f_{0}=20 \mathrm{MHz}$, i.e., $\lambda_{0}=15 \mathrm{~m}$, with negligible leakages and no absorbing materials, we should expect

$$
M_{\mathrm{W}}(\nu)=\frac{1}{2 \pi}+6.37 \cdot 10^{-5} \nu^{2.5},
$$

which can now be evaluated at multiples of the fundamental resonance. We shall consider the two most widely applied thumb rules for overmoded conditions: 1) a frequency about 5 or 10 times $f_{0}$, i.e., $\nu=5$ or $\nu=10 ; 2$ ) a frequency where the cumulative number of modes is $N \geq 60$, for which Weyl's approximation (2) implies

$$
V_{\lambda}=\frac{45}{2 \pi}
$$

corresponding for a cubic cavity to $a / \lambda \simeq 1.92$ and $\nu=2.7$. We obtain $M_{\mathrm{W}}(2.7)=0.16, M_{\mathrm{W}}(5)=0.17$ and $M_{\mathrm{W}}(10)=$ 0.18 . In all of these cases, $M_{\mathrm{W}}<1$, thus making the case for strong fluctuations in the modal density an important issue, as implied by the results shown in Fig. 11 for weak average modal overlapping and discussed in Section VII. Fig. 12 shows $M_{\mathrm{W}}(\nu)$ for a varying frequency, in the case where $\bar{Q}_{3} \rightarrow \infty$ : based on dissipation in the antenna load and finite conductivity over the cavity boundary, a modal overlapping $M_{\mathrm{W}}=1$ is to be expected only above 45 times $f_{0}$.

Clearly, the presence of a lossy EUT or absorbers within the cavity would increase $M_{\mathrm{W}}$. Indeed, for a perfectly absorbing material, the absorption cross-section would be approximately equal to its geometrical cross section. Hence, an additional factor $4 \bar{\sigma}_{\text {eq }} / \lambda^{2}$ should be included and could be expected to be the dominant one around the LUF. This conclusion agrees with the observations made in [13], where it was shown that an unloaded cavity can be incapable of supporting a diffuse-field condition even above the lowest usable frequency defined in the IEC standard [12]. The inclusion of additional losses seems to be necessary in the lower frequency range, for the sake of creating more easily reproducible conditions for the field statistics, i.e., with field statistics approaching the asymptotic ones derived in [2], [30].

\section{REFERENCES}

[1] T. Lehman, "A statistical theory of electromagnetic fields in complex cavities," Interaction Notes, Note 494, 1993.

[2] J. Kostas and B. Boverie, "Statistical model for a mode-stirred chamber," IEEE Transactions on Electromagnetic Compatibility, vol. 33, no. 4, pp. $366-370$, nov 1991.

[3] P. Corona, G. Ferrara, and M. Migliaccio, "Reverberating chambers as sources of stochastic electromagnetic fields," IEEE Transactions on Electromagnetic Compatibility, vol. 38, no. 3, pp. 348 -356, Aug 1996.

[4] G. Orjubin, E. Richalot, S. Mengue, and O. Picon, "Statistical model of an undermoded reverberation chamber," IEEE Transactions on Electromagnetic Compatibility, vol. 48, no. 1, pp. 248 -251, feb. 2006.

[5] L. Arnaut, "Compound exponential distributions for undermoded reverberation chambers," IEEE Transactions on Electromagnetic Compatibility, vol. 44, no. 3, pp. 442 - 457, aug 2002.

[6] - "Limit distributions for imperfect electromagnetic reverberation," IEEE Transactions on Electromagnetic Compatibility, vol. 45, no. 2, pp. 357 - 377, may 2003.

[7] J. Ladbury, G. Koepke, and D. Camel, Evaluation of the NASA Langley Research Center mode-stirred chamber facility, ser. NIST technical note. US Department of Commerce, National Institute of Standards and Technology, 1999, no. 1508.

[8] P. Corona, G. Ferrara, and M. Migliaccio, "Reverberating chamber electromagnetic field in presence of an unstirred component," IEEE Transactions on Electromagnetic Compatibility, vol. 42, no. 2, pp. 111 -115 , may 2000.

[9] O. Lundén and M. Bäckström, "How to avoid unstirred high frequency components in mode stirred reverberation chambers," in Electromagnetic Compatibility, 2007. EMC 2007. IEEE International Symposium on, July 2007, pp. 1-4.

[10] V. Primiani, F. Moglie, and V. Paolella, "Numerical and experimental investigation of unstirred frequencies in reverberation chambers," in Electromagnetic Compatibility, 2009. EMC 2009. IEEE International Symposium on, August 2009, pp. 177-181.

[11] R. Serra and F. Canavero, "Bivariate statistical approach for goodbut-imperfect electromagnetic reverberation," IEEE Transactions on Electromagnetic Compatibility, vol. 53, no. 3, pp. 554 -561, aug. 2011.

[12] Testing and measurement techniques - Reverberation chamber test methods, International Electrotechnical Commission (IEC) Std. 61 0004-21, 2011.

[13] A. Cozza, "The Role of Losses in the Definition of the Overmoded Condition for Reverberation Chambers and Their Statistics," IEEE Transactions on Electromagnetic Compatibility, no. 53, pp. 296-307, 2011.

[14] H. Stöckmann, Quantum chaos: an introduction. Cambridge Univ Pr, 1999, vol. 3.

[15] M. Mehta, Random matrices., ser. Pure and Applied Mathematics. Amsterdam: Elsevier, 2004, vol. 142.

[16] C. Vaa, P. M. Koch, and R. Blümel, "Weyl formula: Experimental test of ray splitting and corner corrections," Phys. Rev. E, vol. 72, p. 056211, Nov 2005

[17] E. Wigner, "Random matrices in physics," Siam Review, vol. 9, no. 1, pp. $1-23,1967$.

[18] H.-J. Stöckmann and J. Stein, "Quantum chaos in billiards studied by microwave absorption," Physical Review Letters, vol. 64, pp. 2215-2218, May 1990.

[19] O. Bohigas, M. Giannoni, and C. Schmit, "Characterization of chaotic quantum spectra and universality of level fluctuation laws," Physical Review Letters, vol. 52, no. 1, pp. 1-4, 1984.

[20] M. Berry and M. Tabor, "Level clustering in the regular spectrum," Proceedings of the Royal Society of London. A. Mathematical and Physical Sciences, vol. 356, no. 1686, pp. 375-394, 1977.

[21] F. Haake, G. Lenz, P. Seba, J. Stein, H. Stöckmann, and K. Życzkowski, "Manifestation of wave chaos in pseudointegrable microwave resonators," Physical Review A, vol. 44, no. 10, pp. 6161-6164, 1991.

[22] G. Orjubin, E. Richalot, O. Picon, and O. Legrand, "Chaoticity of a reverberation chamber assessed from the analysis of modal distributions obtained by FEM," Electromagnetic Compatibility, IEEE Transactions on, vol. 49, no. 4, pp. 762-771, 2007.

[23] A. Papoulis, Stochastic processes. McGraw-Hill, New York, 1965.

[24] J. Pečarić, F. Proschan, and Y. Tong, Convex functions, partial orderings, and statistical applications. Academic Press, 1992, vol. 187.

[25] B. Liu, D. Chang, and M. Ma, "Eigenmodes and the composite quality factor of a reverberating chamber," US Nat. Bur. Stand. Tech. Note, vol. 1066,1983 
[26] M. Schroeder, "Statistical parameters of the frequency response curves of large rooms," J. Audio Eng. Soc, vol. 35, no. 5, pp. 299-305, 1987.

[27] L. Arnaut, "Operation of electromagnetic reverberation chambers with wave diffractors at relatively low frequencies," Electromagnetic Compatibility, IEEE Transactions on, vol. 43, no. 4, pp. 637 -653, nov 2001.

[28] W. Petirsch and A. Schwab, "Investigation of the field uniformity of a mode-stirred chamber using diffusers based on acoustic theory," Electromagnetic Compatibility, IEEE Transactions on, vol. 41, no. 4, pp. 446 -451, nov 1999.

[29] D. Hill, "Electromagnetic Theory of Reverberation Chambers," National Institute of Standards and Technology, Tech. Rep., 1998.

[30] - "Plane wave integral representation for fields in reverberation chambers," IEEE Transactions on Electromagnetic Compatibility, vol. 40, no. 3, pp. 209-217, 1998. 